\title{
Thymic carcinoma outcomes and prognosis: Results of an international analysis
}

\author{
Usman Ahmad, MD, ${ }^{\mathrm{a}}$ Xiaopan Yao, PhD, ${ }^{\mathrm{b}, \mathrm{c}}$ Frank Detterbeck, MD, ${ }^{\mathrm{d}}$ James Huang, MD, ${ }^{\mathrm{a}}$ \\ Alberto Antonicelli, MD, ${ }^{\mathrm{d}}$ Pier Luigi Filosso, MD, ${ }^{\mathrm{e}}$ Enrico Ruffini, MD, ${ }_{\mathrm{e}}^{\mathrm{e}}$ William Travis, MD, \\ David R. Jones, MD, ${ }^{\mathrm{a}}$ Yilei Zhan, MD, ${ }^{\mathrm{b}}$ Marco Lucchi, MD, ${ }^{\mathrm{g}}$ and Andreas Rimner, $\mathrm{MD}^{\mathrm{h}}$
}

Objectives: The objectives of this collaborative study were to characterize patients with thymic carcinoma, their treatment patterns, and association with overall survival (OS) and recurrence-free survival (RFS).

Methods: Clinical, pathologic, treatment, and follow-up information were analyzed. OS and RFS were the primary outcome measures.

Results: In 1042 cases of thymic carcinoma, $42(5 \%)$ patients had pathologic Masaoka stage I, $138(17 \%)$ had stage II, $370(45 \%)$ had stage III, and $274(33 \%)$ had stage IV disease. Overall, 166 patients $(22 \%)$ underwent induction chemotherapy and $48(6 \%)$ had preoperative radiation therapy. An R0 resection was performed in 447 cases $(61 \%)$, R1 in 102 cases (14\%), and R2 in 184 cases $(25 \%)$. Squamous cell carcinoma was the predominant histologic subtype $(\mathrm{n}=560 ; 79 \%)$. Adjuvant chemotherapy was administered to $237(31 \%)$ patients, and 449 $(60 \%)$ received adjuvant radiation therapy. The median OS was 6.6 years $(95 \%$ confidence interval $[\mathrm{CI}], 5.8$ 8.3 ) and the cumulative incidence of recurrence at 5 years was $35 \%(95 \% \mathrm{CI}, 30 \%-40 \%)$. In univariate analysis, early Masaoka stage, $\mathrm{R} 0$ resection, chemotherapy, and radiation therapy were associated with OS. Early Masaoka stage and R0 resection were also associated with RFS. On multivariable analysis, R0 resection and radiation therapy were associated with prolonged OS. Radiation therapy and male gender were associated with prolonged RFS.

Conclusions: R0 resection and radiation therapy are associated with improved OS, whereas radiation therapy and male gender are associated with longer RFS. (J Thorac Cardiovasc Surg 2015;149:95-101)

See related commentary on pages 101-2.

Supplemental material is available online.

Thymic carcinomas are rare thymic neoplasms. ${ }^{1,2}$ Our knowledge of treatment and prognostic factors is limited to small retrospective series. Only 2 multicenter studies

\footnotetext{
From Thoracic Surgery, ${ }^{\text {a }}$ Memorial Sloan Kettering Cancer Center, New York, NY; Department of Biostatistics, ${ }^{\mathrm{b}}$ Medical Oncology, ${ }^{\mathrm{c}}$ Department of Internal Medicine, and Department of Thoracic Surgery, ${ }^{\mathrm{d}}$ Yale University School of Medicine, New Haven, Conn; Department of Thoracic Surgery, ${ }^{\mathrm{e}}$ School of Medicine, University of Torino, Turin, Italy; Department of Pathology, ${ }^{\mathrm{f}}$ Memorial Sloan Kettering Cancer Center, New York, NY; Division of Thoracic Surgery, ${ }^{\mathrm{g}}$ Cardiac and Thoracic Department, Azienda Ospedaliero-Universitaria Pisana, Pisa, Italy; and Department of Radiation Oncology, ${ }^{\mathrm{h}}$ Memorial Sloan Kettering Cancer Center, New York, NY

Disclosures: Authors have nothing to disclose with regard to commercial support.

Read at the 94th Annual Meeting of The American Association for Thoracic Surgery, Toronto, Ontario, Canada, April 26-30, 2014.

Received for publication April 7, 2014; revisions received Sept 26, 2014; accepted for publication Sept 28, 2014

Address for reprints: Andreas Rimner, MD, Department of Radiation Oncology, Memorial Sloan Kettering Cancer Center, 1275 York Ave, New York, NY 10065 (E-mail: rimnera@mskcc.org).

$0022-5223 / \$ 36.00$

Copyright (C) 2015 by The American Association for Thoracic Surgery

http://dx.doi.org/10.1016/j.jtcvs.2014.09.124
}

have been reported so far, including a review of 186 patients from 115 centers in Japan, ${ }^{3}$ and an analysis of 290 cases in the Surveillance, Epidemiology, and End Results (SEER) registry. ${ }^{4}$

In order to study these tumors in a large international sample, the International Thymic Malignancy Interest Group (ITMIG) and the European Society of Thoracic Surgeons (ESTS) created a combined database of all thymic malignancies. ${ }^{5-7}$ Here, we report the first analysis from this combined database of patients with thymic carcinomas, which is the largest analysis of this rare disease to date. The objective of this study was to identify the prognostic, patient, tumor, and treatment characteristics that are associated with overall survival (OS) and recurrence-free survival (RFS) in patients with thymic carcinoma.

\section{METHODS}

Approval for this study was granted by the Yale University Institutional review board (\#1307012419).

\section{Patients}

The ITMIG database was launched in September 2012; further details are described elsewhere. ${ }^{6}$ Cases of thymic carcinoma from 67 participating institutions were included in this analysis. Participating institutions are listed in Appendix E1. The ESTS database was populated in parallel by other participating centers (Appendix E2). The ITMIG and ESTS databases collected limited datasets from which deidentified data were extracted. 


\section{Abbreviations and Acronyms \\ CIR = cumulative incidence of recurrence \\ ESTS = European Society of Thoracic Surgeons \\ IASLC $=$ International Association for the Study of Lung Cancer \\ ITMIG = International Thymic Malignancy Interest Group \\ OS = overall survival \\ RFS = recurrence-free survival \\ SEER $=$ Surveillance, Epidemiology, and End Results}

Multiple rounds of requests for missing data were performed in order to obtain a dataset that was as complete as possible. Despite this effort and the standardization of variables across databases, not all data were retrievable. Therefore the number of cases that could be analyzed for each variable and test are reported.

\section{Clinicopathologic Variables and Outcomes}

Data regarding demographic and clinical characteristics, histology, tumor size and invasion, staging, surgery, chemotherapy, and radiation therapy were collected. Data on recurrence and survival was also collected. For the purpose of this report, only the first recurrence and the associated recurrence-free interval were included.

Time intervals were calculated according to the following descriptions. Overall survival. OS was determined as the time interval between date of surgery, or the last day of therapy if no surgery, and the date of death or the date when last known alive.

Recurrence-free survival. RFS was determined as the time interval between date of surgery or the last day of therapy if no surgery, and the date when recurrence was noted or the date of last follow-up without recurrence.

Staging system. Various centers reported tumor stage using either the Masaoka ${ }^{8}$ or Masaoka-Koga ${ }^{9}$ systems. Initial analyses revealed a lack of difference in outcomes between stages I and II and the ITMIG/International Association for the Study of Lung Cancer (IASLC) staging system. The Prognostic Factors Committee of ITMIG also found no difference in outcomes between stages I and II. ${ }^{10}$ Therefore, cases with stages I and II (II, IIa, IIb) were pooled and analyzed together, and cases staged using the Masaoka and Masaoka-Koga staging systems were combined.

In this cohort, lymph node involvement was reported in only 20 patients. The role of lymph node involvement could not be studied because of the small number of patients with positive nodes and because there is no consistent practice of lymphadenectomy or lymph node sampling. Data on the presence of distant metastases were reported and incorporated into the Masaoka stage.

\section{Statistical Analysis}

A core statistical team of ITMIG investigators (X.Y. and Y.Z.) performed all analyses with SAS 9.3 and R. OS and RFS were the primary outcomes. The association of OS with clinical and prognostic factors was tested using the log-rank test. Prognostic factors that were significantly associated with survival on univariate analysis and clinically relevant factors were included in a Cox proportional hazards model for multivariate analysis.

The cumulative incidence of recurrence (CIR) was assessed using competing risk analysis, with death included as the competing event (curves of death were not shown in cumulative incidence plots). The effect of clinical factors on freedom from recurrence was assessed using Gray's test. The Cox proportional hazards model was used for multivariate analysis.

\section{RESULTS}

Fourteen percent of all thymic tumors in the ITMIG database were thymic carcinomas. A total of 1042 cases of thymic carcinoma were identified for analysis. There was no geographic predominance, and most cases were diagnosed and treated between 2001 and 2010 (Table 1).

The clinical characteristics are described in Table 1. A pretreatment biopsy was performed in $57 \%$ of cases (needle biopsy, 19\%; core biopsy, 10\%; surgical biopsy, 20\%). Two hundred fifty-one $(35 \%)$ cases were assigned a stage using the Masaoka system and 297 (41\%) using the Masaoka-Koga system. A large proportion of patients were upstaged (Table E1). Squamous cell carcinoma was by far the most common histologic subtype (79\%) followed by lymphoepithelioma-like and basaloid histologies (Table 1). There was no significant association between stage at presentation and histologic subtype $(P=.4)$. Surgery, radiation, and chemotherapy treatment details are shown in Tables 2 and E2. Seven hundred thirty-three patients $(70 \%)$ had resection status data available and $447(61 \%)$ had R0 resections (Table 2). Four hundred ninety-four patients $(65 \%)$ received neoadjuvant or adjuvant chemotherapy. Radiation treatment was administered to 545 patients $(72 \%)$ and 449 patients $(82 \%)$ received it in the adjuvant setting. Only 48 patients $(9 \%)$ had radiation as neoadjuvant treatment.

\section{Survival and Recurrence}

Median follow-up in the overall cohort was 4.4 years. Vital status was known for 836 patients $(80 \%)$. Of these, 303 patients $(36 \%)$ had died. The cause of death was available for 224 patients (74\%): 164 deaths $(74 \%)$ were associated with thymic carcinoma, 4\% with treatment complications, and $13 \%$ were attributed to other causes. The median OS for all cases of thymic carcinoma was 6.6 years $(95 \%$ confidence interval, 5.8-8.3). The overall 5 -year survival was $60 \%$ and 10 -year survival was $40 \%$. The overall CIR was $35 \%$ at 5 years and $40 \%$ at 10 years.

\section{Prognostic Factors}

Masaoka stage was significantly associated with OS $(P<.0001$; Figure $1, A)$. The 5 -year OS by stage was $80 \%$ for stage I/II, $63 \%$ for stage III, $42 \%$ for stage IVa, and $30 \%$ for stage IVb. The 10 -year OS was $60 \%$ for stage I/II, $42 \%$ for stage III, $28 \%$ for stage IVa, and $13 \%$ for stage IVb.

Masaoka stage was also significantly associated with CIR $(P=.0005$; Figure $1, B)$. The CIR for stages I and II was $15 \%$ at both 5 and 10 years, but for stage III, the CIR was 
TABLE 1. Demographic and clinical characteristics $(N=1042)$

\begin{tabular}{|c|c|c|}
\hline & $\mathbf{n}$ & $\%$ \\
\hline \multicolumn{3}{|l|}{ Decade of diagnosis $(\mathrm{n}=910)$} \\
\hline $1980-1990$ & 8 & 0.9 \\
\hline $1991-2000$ & 172 & 19 \\
\hline 2001-2010 & 605 & 67 \\
\hline 2011 onward & 125 & 14 \\
\hline \multicolumn{3}{|l|}{ Continent $(\mathrm{n}=1042)$} \\
\hline Europe & 456 & 44 \\
\hline Asia & 328 & 32 \\
\hline North America & 258 & 25 \\
\hline $\operatorname{Age}(y ;$ mean $\pm 1 \mathrm{SD})$ & 885 & $56 \pm 14$ \\
\hline \multicolumn{3}{|l|}{ Gender $(n=1025)$} \\
\hline Male & 624 & 61 \\
\hline Female & 401 & 39 \\
\hline \multicolumn{3}{|c|}{ Paraneoplastic syndromes $(\mathrm{n}=833)$} \\
\hline None & 757 & 91 \\
\hline Myasthenia gravis & 56 & 7 \\
\hline Hypogamma-globulinemia & 1 & 0.1 \\
\hline Red cell aplasia & 1 & 0.1 \\
\hline Other & 18 & 2 \\
\hline \multicolumn{3}{|c|}{ Previous malignancy/second primary $(\mathrm{n}=629)$} \\
\hline None & 551 & 88 \\
\hline Skin & 11 & 1.7 \\
\hline Breast & 11 & 1.7 \\
\hline Lung & 7 & 1 \\
\hline Hematologic & 6 & 1 \\
\hline Colorectal & 3 & 0.5 \\
\hline Prostate & 3 & 0.5 \\
\hline Other & 37 & 6 \\
\hline \multicolumn{3}{|c|}{ Clinical Masaoka stage $(n=408)$} \\
\hline I & 40 & 10 \\
\hline II & 64 & 16 \\
\hline III & 155 & 38 \\
\hline IV-NOS & 4 & 1 \\
\hline IVa & 76 & 19 \\
\hline $\mathrm{IVb}$ & 69 & 17 \\
\hline \multicolumn{3}{|c|}{ Pathologic Masaoka stage $(n=824)$} \\
\hline I & 42 & 5 \\
\hline II & 138 & 17 \\
\hline III & 370 & 45 \\
\hline IV-NOS & 21 & 3 \\
\hline IVa & 114 & 14 \\
\hline $\mathrm{IVb}$ & 139 & 17 \\
\hline \multicolumn{3}{|l|}{ WHO histology $(\mathrm{n}=706)$} \\
\hline Squamous & 560 & 79 \\
\hline Basaloid & 21 & 3 \\
\hline Mucoepidermoid & 18 & 2.5 \\
\hline Lymphoepithelioma-like & 40 & 6 \\
\hline Sarcomatoid & 18 & 2.5 \\
\hline Clear cell & 9 & 1.3 \\
\hline Adenocarcinoma & 11 & 1.6 \\
\hline Undifferentiated & 18 & 2.5 \\
\hline Other & 11 & 1.6 \\
\hline
\end{tabular}

$35 \%$ at 5 years and $38 \%$ at 10 years. The CIR for stages IVa and IVb was $45 \%$ at 5 years and $52 \%$ at 10 years.

We found no association between histologic subtype and either OS or CIR, either in the overall cohort (Figure 2, A and $B$ ) or in the subset of patients who underwent an R0 resection (Figure E1). We were unable to identify a pattern of high-risk or low-risk histologic subtypes.

\section{Treatment Patterns}

An R0 resection was significantly associated with improved OS at 5 years (R0, 70\%; R1, 55\%; R2, 48\%; $P=.0002)$. No significant differences were noted in OS in patients who had $\mathrm{R} 1$ and $\mathrm{R} 2$ resections (Figure $\mathrm{E} 2, A$ ). Among patients who had an R0 resection, pathologic Masaoka stage was significantly associated with OS $(P<.0001)$ (Figure E2, $B)$.

Resection status was also a significant predictor for RFS in the competing risk analysis $(P=.0124$, Figure E2, $C)$. The CIR for R0 was $34 \%$ at 5 years and $38 \%$ at 10 years, but for R1/2, the CIR was $45 \%$ at 5 years and $49 \%$ at 10 years.

Chemotherapy was not administered to most patients with stage I (58\%) and II (66\%) thymic carcinoma; $65 \%$ of patients with stage III thymic carcinoma did receive chemotherapy (neoadjuvant, 19\%; adjuvant, 37\%). For both stage IVa and IVb, approximately $30 \%$ of patients received chemotherapy in both neoadjuvant and adjuvant settings.

Radiation therapy was delivered to more than $70 \%$ of patients with the exception of patients with stage I thymic carcinoma $(55 \%)$. Most patients underwent radiation therapy in the adjuvant setting. Overall, the most common treatment patterns of adjuvant modalities were adjuvant chemotherapy and radiation (26\%) and adjuvant radiation only $(22 \%)$. A combination of neoadjuvant chemotherapy and adjuvant radiation therapy was used in $10 \%$ of patients. Overall, $15 \%$ of patients received neither chemotherapy nor radiation (Table 2).

\section{Impact of Multimodality Treatment}

Both chemotherapy $(P=.0004)$ and radiation therapy $(P=.0006)$ were associated with significant clinical benefit and prolonged OS. However, because of the limited numbers available in each subgroup, we were unable to refine whether the multimodality treatment pattern that was associated with improved OS was in the definitive, neoadjuvant, or adjuvant setting. In univariable analysis, neither chemotherapy $(P=.256)$ nor radiation therapy $(P=.602)$ had any association with RFS.

On multivariate analysis, $\mathrm{R} 0$ resection $(P=.0071)$ and the use of any radiation treatment $(P=.0055)$ were 
TABLE 2. Use of treatment modalities

\begin{tabular}{|c|c|c|c|c|c|c|c|}
\hline & $\mathbf{n}$ & $\%$ & Stage I $(\%)$ & Stage II $(\%)$ & Stage III (\%) & Stage IVA $(\%)$ & Stage IVB $(\%)$ \\
\hline Surgical resection & 733 & & $\mathrm{n}=38$ & $\mathrm{n}=112$ & $\mathrm{n}=311$ & $\mathrm{n}=97$ & $\mathrm{n}=94$ \\
\hline R0 & 447 & 61 & 95 & 85 & 64 & 46 & 43 \\
\hline $\mathrm{R} 1$ & 102 & 14 & 2.6 & 14 & 14 & 14 & 19 \\
\hline $\mathrm{R} 2$ & 184 & 25 & 2.6 & 1 & 22 & 39 & 38 \\
\hline Chemotherapy & 764 & & $\mathrm{n}=38$ & $\mathrm{n}=111$ & $\mathrm{n}=310$ & $\mathrm{n}=105$ & $\mathrm{n}=115$ \\
\hline No chemotherapy & 270 & 35 & 58 & 66 & 35 & 20 & 21 \\
\hline Neoadjuvant & 166 & 22 & 5 & 14 & 19 & 32 & 26 \\
\hline Adjuvant & 237 & 31 & 34 & 18 & 37 & 27 & 36 \\
\hline Before and after resection & 60 & 8 & 3 & 2 & 9 & 13 & 6 \\
\hline Definitive/palliative & 31 & 4 & 0 & 1 & 1 & 8 & 11 \\
\hline Radiation & 754 & & $\mathrm{n}=38$ & $\mathrm{n}=116$ & $\mathrm{n}=303$ & $\mathrm{n}=103$ & $\mathrm{n}=112$ \\
\hline No radiotherapy & 209 & 28 & 55 & 27 & 23 & 31 & 31 \\
\hline Neoadjuvant & 48 & 6 & 0 & 6 & 4 & 7 & 9 \\
\hline Adjuvant & 449 & 60 & 42 & 65 & 67 & 52 & 50 \\
\hline Before and after resection & 29 & 4 & 0 & 2 & 5 & 4 & 3 \\
\hline Definitive/palliative & 19 & 2.5 & 3 & 1 & 0.3 & 7 & 7 \\
\hline
\end{tabular}

significantly associated with improved OS (Table 3). Radiation treatment $(P=.0090)$ and male gender $(P=.0122)$ were significantly associated with improved RFS (Table 4).

\section{DISCUSSION}

In this retrospective database study, we found early Masaoka stage, R0 resection, and multimodality treatment with surgery and radiation to be associated with prolonged OS. In addition, radiation treatment was associated with prolonged RFS.

Lee and colleagues ${ }^{11}$ previously reported early Masaoka stage (I and II) compared with advanced stage (III and IV) to be significantly associated with improved survival. They did not observe a difference in OS between stages III and IV. Masaoka stage was also significantly associated with OS in the SEER database analysis. ${ }^{4}$ Similarly, Kondo and colleagues ${ }^{3}$ reported a significant difference in OS between stages I/II and III/IV. Masaoka stage seems to be the most consistent prognostic factor across various studies.

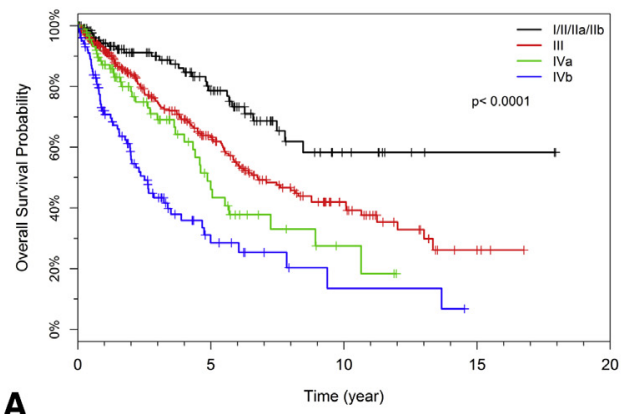

FIGURE 1. Association of pathologic Masaoka stage and resection status with overall survival and cumulative incidence of recurrence. A, Overall survival by pathologic stage in all patients with thymic carcinoma regardless of resection status. B, Cumulative incidence of recurrence by pathologic stage in all patients with thymic carcinoma regardless of resection status.

Complete surgical resection has been reported to be an important prognostic factor. The rate of complete resection varies from $20 \%$ to $88 \%$ in published series. ${ }^{3,411-14}$ In this database, $61 \%$ of cases underwent an $\mathrm{R} 0$ resection and the rate of complete resection decreased with increasing stage. Patients who underwent an R0 resection had a higher 5-year $(70 \%$ vs $55 \%)$ and 10 -year $(50 \%$ vs $35 \%)$ survival compared with patients who had an R1/2 resection. It therefore seems that a complete resection may still lead to long-term RFS even in locally advanced thymic carcinomas, and an R0 resection should therefore be the goal of any surgical approach. This is primarily a surgical series and our database did not capture many patients treated nonsurgically.

There are limited data about the role of radiation therapy in the treatment of thymic carcinoma. We found that most patients at any stage underwent postoperative radiation. On multivariate analysis, the use of radiation treatment was the strongest variable associated with survival. Ogawa and colleagues ${ }^{13}$ reported no local recurrence in 16 patients treated with complete resection and adjuvant radiation, and

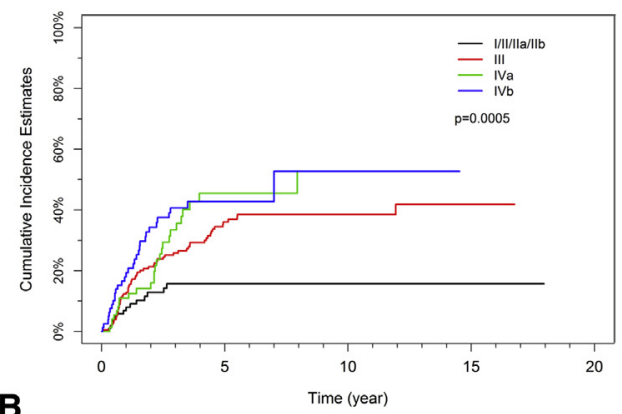



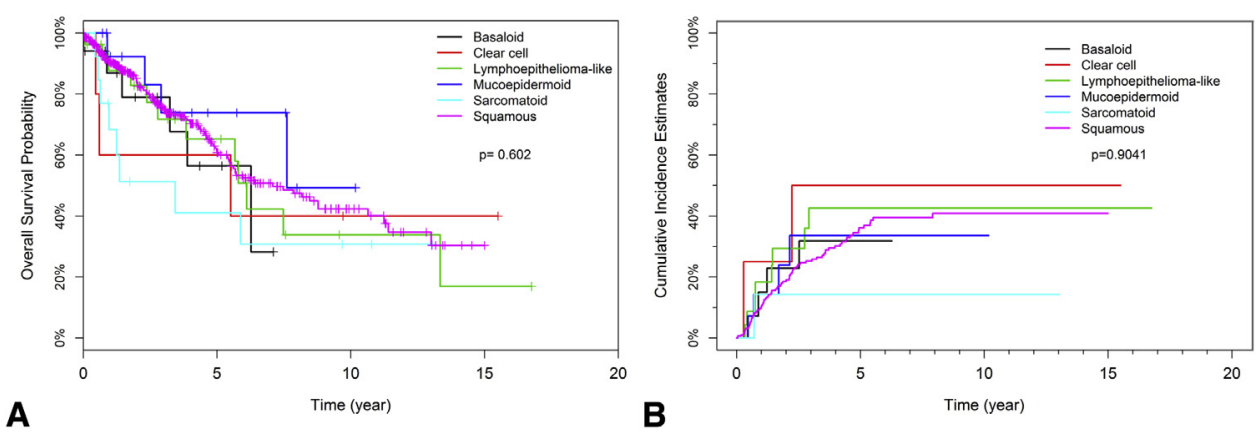

FIGURE 2. Association of histologic subtype with overall survival and cumulative incidence of recurrence. A, Overall survival by histologic subtype in all patients. B, Cumulative incidence of recurrence by histologic subtype in all patients.

recommended adjuvant radiation. Kondo and colleagues ${ }^{3}$ reported improved OS with adjuvant chemoradiotherapy but no difference in recurrence with the use of adjuvant radiation treatment. Two other reports, however, did not find postoperative radiation to be statistically associated with survival. ${ }^{4,15}$ Most investigators recommend an aggressive multimodal approach including complete resection and postoperative radiation in the management of thymic carcinomas. Our findings suggest that adjuvant radiation therapy is often used in patients with thymic carcinoma and seems to be associated with a significant improvement on OS and RFS. Chemotherapy was not associated with OS or RFS in multivariate analysis. This is different from previous reports where the use of chemotherapy was noted to be beneficial in surgical ${ }^{16}$ and nonsurgical ${ }^{17}$ medical regimens.

In this series, squamous cell carcinoma was the most common histology, as previously reported $(62 \%-70 \%))^{3,14,17}$

TABLE 3. Multivariate analysis of prognostic and treatment variables associated with OS

\begin{tabular}{lcc}
\hline \multicolumn{1}{c}{ Parameter } & $\begin{array}{c}\text { Hazard ratio } \\
\text { (95\% confidence interval) }\end{array}$ & $\boldsymbol{P}$ value* \\
\hline Pathologic stage & $1.287(0.556,2.983)$ & .1760 \\
III (vs I/II/IIa/IIb) & $1.483(0.561,3.921)$ & $.5558 \dagger$ \\
IVa (vs I/II/IIa/IIb) & $2.428(0.983,5.995)$ & $.4274 \dagger$ \\
IVb (vs I/II/IIa/IIb) & $2.211(1.241,3.940)$ & $.0544 \dagger$ \\
Resection status & & .0071 \\
$\quad$ R1/2 (vs R0) & $1.536(0.828,2.848)$ & .1732 \\
Chemotherapy & & \\
$\quad$ Yes (vs no) & $0.454(0.260,0.793)$ & .0055 \\
Radiation & & \\
$\quad$ Yes (vs no) & $1.542(0.904,2.631)$ & .1122 \\
Gender & & \\
$\quad$ Female (vs male) & $0.780(0.391,1.557)$ & .4815 \\
Histology & & .2957 \\
$\quad$ Squamous (vs others) & & .5800 \\
Myasthenia gravis & $0.557(0.186,1.668)$ & \\
$\quad$ Yes (vs no) & $0.978(0.904,1.058)$ & \\
Tumor size &
\end{tabular}

*Likelihood ratio $P$ value from a Cox model. †Wald $P$ values.
There were no significant differences in clinical characteristics between the histologic subtypes. In addition, the histologic subtypes had no association with stage at presentation, recurrence, or OS. Previously, histologic subtypes have been categorized as high or low risk, ${ }^{18}$ however, no clear prognostic benefit has been shown in patients treated medically ${ }^{17}$ or surgically. ${ }^{19}$ This is corroborated by our inability to detect any difference in outcomes between different histologic subtypes.

Only $40 \%$ of the patients had a clinical stage assigned or available. A significant rate of stage shifting was noted. This suggests the need for improved clinical staging methods as well as systemic application of staging techniques.

Our study represents the largest series of thymic carcinoma to date. Because of the extent and breadth of this global collaboration, it is unlikely that more data about patients treated during this time period can be amassed.

TABLE 4. Multivariate analysis of prognostic and treatment variables associated with recurrence-free survival

\begin{tabular}{lcc}
\hline \multicolumn{1}{c}{ Parameter } & $\begin{array}{c}\text { Hazard ratio } \\
(\mathbf{9 5} \% \text { confidence interval })\end{array}$ & $\boldsymbol{P}$ value* \\
\hline Pathologic stage & $1.767(0.849,3.676)$ & .1215 \\
$\quad$ III (vs I/II/IIa/IIb) & $2.601(1.136,5.955)$ & $.1279 \dagger$ \\
$\quad$ Iva (vs I/II/IIa/IIb) & $2.295(0.986,5.343)$ & $.0237 \dagger$ \\
IVb (vs I/II/IIa/IIb) & $1.566(0.948,2.587)$ & $.071 \dagger$ \\
Resection status & & \\
$\quad$ R1/2 (vs R0) & $1.411(0.828,2.404)$ & .2059 \\
Chemotherapy & & \\
$\quad$ Yes (vs no) & $0.529(0.328,0.853)$ & .0090 \\
Radiation & & \\
$\quad$ Yes (vs no) & $1.853(1.144,3.001)$ & .0122 \\
Gender & \\
$\quad$ Female (vs male) & $1.017(0.575,1.802)$ & .9526 \\
Histology & & \\
$\quad$ Squamous (vs others) & $1.412(0.620,3.215)$ & .4114 \\
Myasthenia gravis & $0.987(0.919,1.061)$ & .7304 \\
$\quad$ Yes (vs no) &
\end{tabular}

*Likelihood ratio $P$ value from a Cox model. †Wald $P$ values. 
Because of the rarity of these tumors, we have to rely on such collaborative series to advance our knowledge of these tumors. In addition to providing a strong base for thymic tumor research, this database has also nurtured international collaborations and has set the background for future research efforts.

The major limitations of this analysis are its retrospective nature, lack of data auditing, variability in treatment and follow-up, and missing data from various centers despite all our efforts. In addition, lack of central pathology review is a potential confounding factor. Most of the data were submitted by surgeons and pathologists, which explains the lack of patients treated nonsurgically in this series. Nevertheless, this is consistent with the generally recommended approach of surgical resection as the cornerstone of thymic carcinoma management whenever possible.

In conclusion, most thymic carcinomas present at advanced stages. Early Masaoka stage, complete resection, and the use of radiation therapy are significantly associated with prolonged OS and RFS. We were unable to show an association of histologic subtypes with significant differences in OS. To move forward in the treatment of thymic carcinoma, it is important to study these patients in a prospective fashion. In addition, maintaining some degree of uniformity in treatment will be helpful in deriving meaningful conclusions about outcomes. Based on these data, we recommend an aggressive surgical approach for potentially resectable tumors followed by adjuvant radiation treatment.

\section{References}

1. de Jong WK, Blaauwgeers JL, Schaapveld M, Timens W, Klinkenberg TJ, Groen HJ. Thymic epithelial tumours: a population-based study of the incidence, diagnostic procedures and therapy. Eur J Cancer. 2008;44: 123-30.

2. Greene MA, Malias MA. Aggressive multimodality treatment of invasive thymic carcinoma. J Thorac Cardiovasc Surg. 2003;125:434-6.

3. Kondo K, Monden Y. Therapy for thymic epithelial tumors: a clinical study of 1320 patients from Japan. Ann Thorac Surg. 2003;76:878-84.

4. Weksler B, Dhupar R, Parikh V, Nason KS, Pennathur A, Ferson PF. Thymic carcinoma: a multivariate analysis of factors predictive of survival in 290 patients. Ann Thorac Surg. 2013;95:299-303.

5. Detterbeck FC. The International Thymic Malignancy Interest Group. J Natl Compr Canc Netw. 2013;11:589-93.

6. Huang J, Ahmad U, Catlin A, Detterbeck FC; on behalf of the ITMIG International Database Committee. Development of the International Thymic Malignancy Interest Group international database: An unprecedented resource for the study of a rare tumor. J Thorac Oncol. 2014;9:1573-8.

7. Ruffini E, Detterbeck F, Van Raemdonck D, Rocco G, Thomas P, Weder W, et al; on behalf of the European Society of Thoracic Surgeons (ESTS) Thymic Working Group. Tumours of the thymus: a cohort study of prognostic factors from the European Society of Thoracic Surgeons database. Eur J Cardiothorac Surg. 2014;46:361-8.

8. Masaoka A, Monden Y, Nakahara K, Tanioka T. Follow-up study of thymomas with special reference to their clinical stages. Cancer. 1981;48: 2485-92.

9. Koga K, Matsuno Y, Noguchi M, Mukai K, Asamura H, Goya T, et al. A review of 79 thymomas: modification of staging system and reappraisal of conventional division into invasive and non-invasive thymoma. Pathol Int. 1994;44:359-67.
10. Detterbeck FC, Asamura H, Crowley J, Falkson C, Giaccone G, Giroux D, et al; Staging and Prognostic Factors Committee; Members of the Advisory Boards; Participating Institutions of the Thymic Domain. The IASLC/ITMIG thymic malignancies staging project: development of a stage classification for thymic malignancies. J Thorac Oncol. 2013;8:1467-73.

11. Lee CY, Bae MK, Park IK, Kim DJ, Lee JG, Chung KY. Early Masaoka stage and complete resection is important for prognosis of thymic carcinoma: a 20-year experience at a single institution. Eur J Cardiothorac Surg. 2009;36: $159-62$.

12. Okereke IC, Kesler KA, Freeman RK, Rieger KM, Birdas TJ, Ascioti AJ, et al. Thymic carcinoma: outcomes after surgical resection. Ann Thorac Surg. 2012; 93:1668-72.

13. Ogawa K, Toita T, Uno T, Fuwa N, Kakinohana Y, Kamata M, et al. Treatment and prognosis of thymic carcinoma: a retrospective analysis of 40 cases. Cancer. 2002;94:3115-9.

14. Hosaka Y, Tsuchida M, Toyabe S, Umezu H, Eimoto T, Hayashi J. Masaoka stage and histologic grade predict prognosis in patients with thymic carcinoma. Ann Thorac Surg. 2010;89:912-7.

15. Hsu HC, Huang EY, Wang CJ, Sun LM, Chen HC. Postoperative radiotherapy in thymic carcinoma: treatment results and prognostic factors. Int J Radiat Oncol Biol Phys. 2002;52:801-5.

16. Lucchi M, Ambrogi MC, Duranti L, Basolo F, Fontanini G, Angeletti CA, et al. Advanced stage thymomas and thymic carcinomas: results of multimodality treatments. Ann Thorac Surg. 2005;79:1840-4.

17. Okuma Y, Hosomi Y, Takagi Y, Sasaki E, Hishima T, Maeda Y, et al. Clinical outcomes with chemotherapy for advanced thymic carcinoma. Lung Cancer. 2013;80:75-80.

18. Suster S, Rosai J. Thymic carcinoma. A clinicopathologic study of 60 cases. Cancer. 1991;67:1025-32.

19. Yano M, Sasaki H, Yokoyama T, Yukiue H, Kawano O, Suzuki S, et al. Thymic carcinoma: 30 cases at a single institution. Thorac Oncol. 2008;3:265-9.

\section{Discussion}

Dr Shaf Keshavjee (Toronto, Ontario, Canada). Congratulations on a very nice presentation. I think the value of this paper lies in being able to speak about results relating to over 1000 cases of thymic carcinoma from 67 institutions and being able to really show survival curves by Masaoka stage for the thymic carcinomas, which in most single-center series get excluded because they are different from other thymic tumors and the numbers are very small.

You have pointed out the limitations of your study, one being that treatment varied from center to center and you cannot really control for that. I think it is fantastic that ITMIG and the ESTS have got together. Actually, I should also disclose a conflict in that the Toronto General Hospital data are included in this dataset, but I was not involved in the writing or analysis of the data. How would you improve on the data that you currently have in terms of really being able to learn from this multicenter data, which have the disadvantage of being so variable in how they are collected and how patients are treated?

Dr Ahmad. Thank you. I think there are several points that I would like to make. First of all, if you look at all these retrospective studies, they originated from institutions that have different treatment patterns and they have different outcomes. Now, we cannot control for the treatments; however, we are looking at the same outcomes. So we have homogenized the data to some extent. Second, I should say that we were enthused by the fact that the international community is so upcoming about these collaborations and it is very encouraging that this international collaboration has really set up the infrastructure for looking at these tumors in a prospective fashion, and I think that is the way to go for these rare tumors. 
Dr Keshavjee. I have one other question. When you talk about $\mathrm{R} 0$ versus $\mathrm{R} 1$ and $\mathrm{R} 2$ resection and the difficulty in showing a difference between $\mathrm{R} 1$ and $\mathrm{R} 2$ resection, from your knowledge of the database, do we have enough information to say, whether you did an R0 resection by doing advanced surgery, like an SVC resection or something like that, that you have a better result than having R1 disease left behind in terms of what we do for our patients, because sometimes when you look at aggregate data, you might give surgeons an excuse to just say, "Well, yes, I'll just sort of debulk this tumor and radiate it," and is that okay or is that inferior surgery?

Dr Ahmad. Well, I think based on the data that we have, what I can say safely is that every effort should be made to achieve an R0 resection and there is really no substitute for an R0 resection.

Dr Keshavjee. That is current dogma, but your data that you showed did not reflect that, right? That is what I think is the danger in looking at data like this, that it did not reflect the dogma that you believe is correct. I believe it, too, but I am just saying that that might be an erroneous interpretation of the data.

Dr Ahmad. Yes, I agree.

Dr Keshavjee. Thank you.

Dr Thomas K. Waddell (Toronto, Ontario, Canada). I will follow up on the point that you just raised because I must say that I did not quite understand what you meant by that. The R1 survival was the same as R2, so my interpretation was that it means that unless you get all of it out, there is no point in doing any surgery. Is that the point you were trying to discuss? I think we do not know what happens to anybody who has no surgery.

Dr Ahmad. Exactly. I think that is what I should clarify some more. You do not have nonsurgically treated patients in the database, so it is hard to compare. If you leave tumor behind or not do surgery at all, how are they going to do in comparison with medical therapy alone? However, historically, if you look at medically treated patients only, their survivals are anywhere between $20 \%$ and $30 \%$, as opposed to R1-resected patients in this series where we had survivals up to $40 \%$.

Dr Anthony J. Acinapura (Brooklyn, New York). Your data suggested that adjuvant therapy is an important factor in overall survival. Some of the more recent publications are suggesting neoadjuvant chemoradiation therapy is equally important and has also been able to allow for more R0 resections. Do you have any data on that?

Dr Ahmad. We were actually unable to show whether adjuvant or neoadjuvant treatment had any benefit. In fact, any chemotherapy or any treatment ended up improving survival. So at this point, based on these data, it is hard for me to conclude that adjuvant therapy itself had any association with overall survival. All we can say is that chemotherapy and radiation helped improve overall survival.

\title{
EDITORIAL COMMENTARY
}

\section{Rarest of a rare disease: Challenges in advancing our knowledge of thymic carcinoma}

\author{
Thomas W. Rice, MD, ${ }^{\mathrm{a}, \mathrm{b}}$ and Eugene H. Blackstone, $\mathrm{MD}^{\mathrm{a}, \mathrm{b}}$
}

See related article on pages 95-101.

Thymic epithelial tumors, with a reported incidence of 3.2 per $1,000,000^{1}$ and thus affecting less than 200,000 people in the United States, are classified as a rare disease by the National Institutes of Health (http://rarediseases. info.nih.gov). The inclusion of rare diseases in the Orphan

\footnotetext{
From the Cleveland Clinic Lerner College of Medicine, ${ }^{\text {a }}$ Cleveland, Ohio; Heart and Vascular Institute, ${ }^{\mathrm{b}}$ Department of Thoracic and Cardiovascular Surgery, Cleveland Clinic, Cleveland, Ohio.

Disclosures: Authors have nothing to disclose with regard to commercial support. Received for publication Nov 11, 2014; accepted for publication Nov 11, 2014.

Address for reprints: Thomas W. Rice, MD, Department of Thoracic and Cardiovascular Surgery, Cleveland Clinic, 9500 Euclid Ave/Desk JJ-4, Cleveland, OH 44195 (E-mail: ricet@ccf.org).

J Thorac Cardiovasc Surg 2015;149:101-2

$0022-5223 / \$ 36.00$

Copyright (c) 2015 by The American Association for Thoracic Surgery http://dx.doi.org/10.1016/j.jtcvs.2014.11.018
}

Drug Act of 1983 has led to the alternative name "orphan diseases." Thymic carcinoma is a subset of thymic epithelial tumors, having approximately one tenth the incidence of all thymic epithelial malignancies. ${ }^{1}$ Thus, the difficulties of acquiring and increasing our knowledge of this orphan disease are confounded by the exceptional rarity of this rarest of a rare disease and the retrospective acquisition of most of the information concerning thymic carcinoma. An understanding of some of these difficulties may enhance the readers' understanding and permit a more focused interpretation of the current article prompting this commentary. ${ }^{2}$

\section{DATA SET AND ANALYSIS}

The planned inclusion of thymic malignancies in the 8th edition of the AJCC Cancer Staging Manual was the impetus to acquire data concerning thymic epithelial tumors, which in turn was the stimulus for formation of the 


\section{APPENDIX E1. LIST OF PARTICIPATING ITMIG CENTERS}

1. University of Padua, Padua, Italy

2. Shanghai Chest Hospital, Shanghai, China

3. Sichuan Cancer Hospital, Chengdu, China

4. Tianjin Cancer Hospital, Tianjin, China

5. Shanghai Pulmonary Disease Hospital, Shanghai, China

6. Beijing Cancer Hospital, Beijing, China

7. Henan Cancer Hospital, Zhengzhou, China

8. Regina Elena National Cancer Institute, Rome, Italy

9. University of Chicago, Chicago, Ill

10. Università Degli Studi di Napoli Federico II, Naples, Italy

11. Maastricht University Medical Centre, Maastricht, Netherlands

12. University Hospitals Leuven, Belgium

13. Massachusetts General Hospital, Boston, Mass

14. Guy's and St Thomas' Hospital, London, United Kingdom

15. Memorial Sloan Kettering Cancer Center, New York, NY

16. University Medical Center Mannheim, Mannheim, Germany

17. Stanford University, Stanford, Calif

18. AHEPA University Hospital, Thessaloniki, Greece

19. Penn Presbyterian Medical Center, Philadelphia, $\mathrm{Pa}$

20. Rigshospitalet, University Hospital of Copenhagen, Denmark

21. Fundeni Clinical Institute, Bucharest, Romania

22. Louis Pradel Hospital, Lyon, France

23. Severance Hospital, Seoul, Korea

24. Azienda Ospedaliero-Universitaria Policlinico V. Emanuele, Catania, Italy

25. Swedish Cancer Institute, Seattle, Wa

26. Fox Chase Cancer Center, Philadelphia, $\mathrm{Pa}$

27. Thoracic Surgery, Azienda Ospedaliera S. Croce e Carle, Cuneo, Italy

28. Hackensack University Medical Center, Hackensack, $\mathrm{NJ}$

29. Mayo Clinic Rochester, Rochester, Minn

30. Hospital Mutua de Terrassa, Terrassa, Spain

31. MD Anderson, Houston, Tex

32. Gangnam Severance Hospital, Seoul, Korea

33. Istanbul University Medical School, Istanbul, Turkey

34. Klinik Schillerhoehe, Gerlingen, Germany

35. Ospedali Riuniti, Ancona, Italy

36. Royal Brompton Hospital/Harefield NHS Foundation Trust, London, United Kingdom

37. Seoul National University Hospital, Seoul, Korea

38. Birmingham Heartlands Hospital, Birmingham, United Kingdom

39. University of Pisa, Pisa, Italy
40. Indiana University Simon Cancer Center, Indianapolis, Ind

41. Yale Cancer Center, New Haven, Conn

42. Oregon Health and Science University, Portland, Ore

\section{APPENDIX E2. LIST OF PARTICIPATING ESTS CENTERS}

1. King Faisal Specialist Hospital, Alfaisal University, Riyadh, Saudi Arabia

2. University of Eastern Piedmont, Novara, Italy

3. Ospedali Riuniti, Ancona, Italy

4. University of Alabama at Birmingham, Birmingham, Ala

5. University of Parma, Parma, Italy

6. General Regional Hospital, Aosta, Italy

7. Aristotle University of Thessaloniki, A.H.E.P.A. University Hospital, Thessaloniki, Greece

8. Uludag University School of Medicine, Bursa, Turkey

9. Hospital Universitario Puerta de Hierro Majadahonda, Madrid, Spain

10. University of Texas, Southwestern Medical Center and School of Medicine, Dallas, Tex

11. Medical University of Vienna, Vienna, Austria

12. IRCCS-CROB Centro Riferimento Oncologico della Basilicata, Rionero in Vulture, Italy

13. Royal Brompton and Harefield NHS Foundation Trust and National Heart and Lung Division, Imperial College, London, United Kingdom

14. Centre Hospitalier de l'Universitè de Montreal, University of Montreal, Montreal, Canada

15. Guy's Hospital, London, United Kingdom

16. SS Antonio e Biagio e Cesare Arrigo Hospital, Alessandria, Italy

17. IRCCS Fondazione Cà Granda Ospedale Maggiore Policlinico, Milan, Italy

18. University Hospital of Salamanca, IBSAL, Salamanca, Spain

19. Hôpital Nord-Aix-Marseille University, Marseille, France

20. University of Torino and AO Città della Salute e della Scienza di Torino, Turin, Italy

21. Vittorio Emanuele Hospital, Catania, Italy

22. Thoracic Surgery, Sacred Heart Hospital Negrar, Verona, Italy

23. Papworth Hospital NHS Foundation Trust, Papworth Everard, Cambridge, United Kingdom

24. University of Rome Sapienza, Policlinico Umberto I, Fondazione Eleonora Lorilard Spencer Cenci, Rome, Italy

25. University Hospitals, Leuven, Belgium

26. Cenci, Rome, Italy

27. University Hospital of Siena, Siena, Italy

28. Medical University of Gdansk, Gdansk, Poland

29. Toronto General Hospital, Toronto, Ontario, Canada 

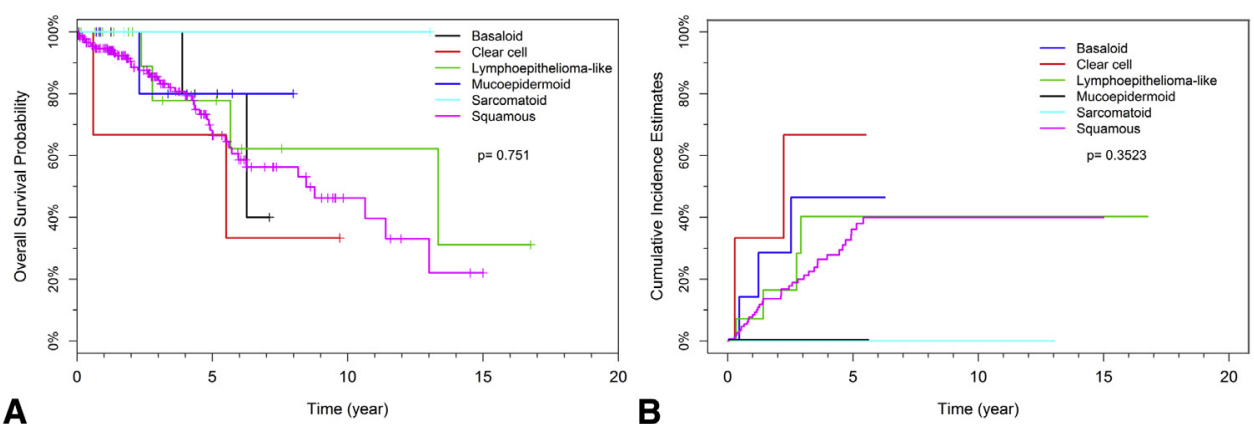

FIGURE E1. Association of histologic subtype with overall survival and cumulative incidence of recurrence in R0 patients. A, Overall survival by histologic subtype in R0 resected patients only. B, Cumulative incidence of recurrence by histologic subtype in R0 resected patients only.
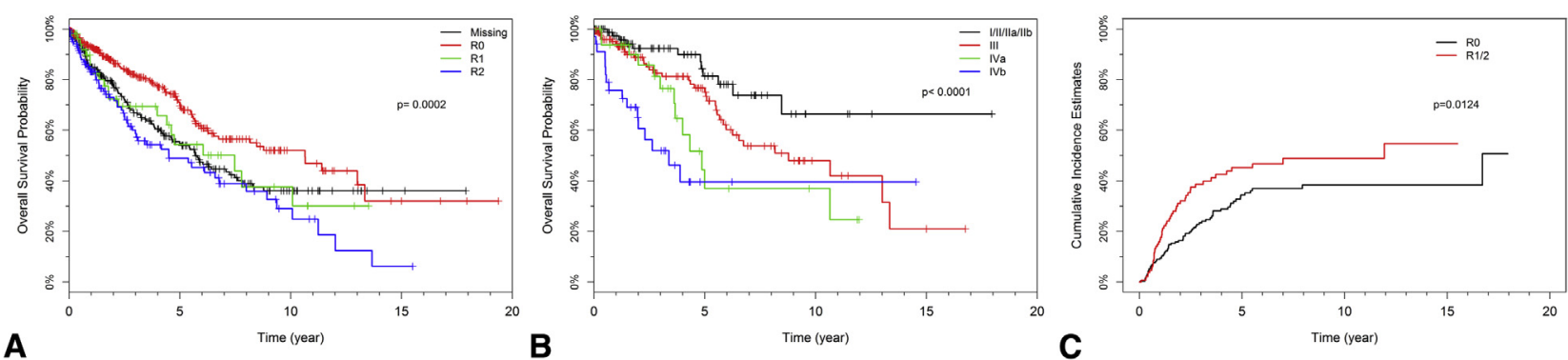

FIGURE E2. Association of pathologic resection status with overall survival. A, Overall survival by resection status in all pathologic stages. B, Overall survival by pathologic stage in R0 resected patients only. C, Recurrence-free survival by resection status in all pathologic stages.

TABLE E2. Surgical approach and tumor size

TABLE E1. Patterns of shift from clinical to pathologic stage $(\mathbf{N}=\mathbf{3 7 0})$

\begin{tabular}{lrrrrrr}
\hline & \multicolumn{5}{c}{ Pathologic stage (\%) } \\
\cline { 2 - 7 } Clinical stage (\%) & I & II & III & IVA & IVB & Total \\
\hline I & 41 & 39 & 13 & 2.6 & 5 & 39 \\
II & 13 & 38 & 44 & 1.6 & 3 & 61 \\
III & 2 & 8 & 76 & 10 & 5 & 146 \\
IVa & 0 & 0 & 9 & 80 & 11 & 66 \\
IVb & 0 & 1.7 & 5 & 1.7 & 90 & 58 \\
\hline
\end{tabular}

$\mathbf{n}$ $\%$ Surgical approach $(\mathrm{n}=624)$

$\begin{array}{lrrr}\text { Sternotomy } & 433 & 69 & 6.2 \pm 3 \\ \text { Thoracotomy } & 79 & 13 & 7 \pm 3 \\ \text { Sternotomy + thoracotomy } & 17 & 3 & 8 \pm 4 \\ \text { Hemi-clamshell } & 26 & 4 & 7 \pm 2 \\ \text { Clamshell } & 8 & 1.3 & 10 \pm 4 \\ \text { VATS } & 39 & 6 & 3.7 \pm 2 \\ \text { VATS-robotic } & 12 & 2 & 4.6 \pm 3 \\ \text { Cervical } & 1 & 0.2 & -\end{array}$

VATS, Video-assisted thoracoscopic surgery. 